\title{
Outbreak of ED visits related to the use of synthetic cannabinoids, Mayotte Island
}

\author{
Pascal Vilain*1, Salamata Bah-Assoumani² ${ }^{2}$ Ali-Mohamed Youssouf ${ }^{2}$ and \\ Laurent Filleul ${ }^{1}$
}

${ }^{1}$ French national public health agency, Regional unit Indian Ocean, Sain-Denis, Réunion; ${ }^{2}$ Hospital center of Mayotte, Mamoudzou, Mayotte

\section{Objective}

To confirm and to characterize the increase in emergency department (ED) visits related to the use of synthetic cannabinoids (SC)

\section{Introduction}

On October 2016, the Indian Ocean Regional Health Agency was alerted about an increase in ED visits related to adverse reactions associated with use of SC on Mayotte Island. In this context, an investigation based on a syndromic surveillance system was implemented by the regional unit of the French national public health agency.

\section{Methods}

An extraction of anonymized records routinely collected by the syndromic surveillance system (1) was carried out from January 1st, 2012 to October 30,2016. ED visits related to the consumption of $\mathrm{SC}$ were identified from ICD-10 codes of the principal diagnostic according to two levels of confidence:

- a probable case was defined as ED visit coded X69 (Intentional self-poisoning by and exposure to other and unspecified chemicals and noxious substances). This code has been implemented specifically by ED physicians since august 2015;

- a suspect case was defined as ED visit coded: F11 (Mental and behavioral disorders due to use of opioids), F12 (Mental and behavioral disorders due to use of cannabinoids), F16 (Mental and behavioral disorders due to use of hallucinogens), F18 (Mental and behavioral disorders due to use of volatile solvents), F19 (Mental and behavioral disorders due to multiple drug use and use of other psychoactive substances).

Based on these data, an epidemic curve and a descriptive analysis of ED visits were carried out.

\section{Results}

In total, $146 \mathrm{ED}$ visits related to adverse events associated with use of SC were registered from January 1 ${ }^{\text {st }}, 2012$ to October 30, 2016. The epidemic curve shows two waves between 2015 and 2016 with a particularly high peak in August 2015 (Figure 1). In total, $49 \%(n=72 / 146)$ of these ED visits were probably related to adverse reactions associated to use $\mathrm{SC}$ and $51 \%(\mathrm{n}=74 / 146)$ meet to the suspect case definition. On the surveillance period, men represented $84 \%$ of the patients $(n=122)$ and median age ( $\min -\max )$ was $23(8-$ $62)$ years old. When the severity score variable was filled $(n=138)$, a vital emergency was reported for $4 \%(n=5)$ of patients and $19 \%$ of patients were hospitalized.

\section{Conclusions}

Data from syndromic surveillance system allowed to confirm an increase in ED visits related to adverse reactions associated with use of SC in Mayotte Island. To our knowledge, it's the first time that an outbreak related to use SC is described in the Ocean Indian areaThis phenomenon was particularly marked in 2015 with a peak of ED visits on August 2016.
After this outbreak, the regional unit of the French national public health agency recommended the pursuit of the coding X69 in principal diagnosis with the following case definition: any patient with an adverse reaction attributed to synthetic cannabinoid use whether suspected by the medical team or declared by the patient himself or if the patient is in possession of the substance; and to raise awareness ED physicians to the notification of these poisonings to the Regional Addictive Surveillance Center.

In conclusion, the young population, weakened by a precarious socio-economic situation, is a target for new synthetic drugs and a threat to public health. This emerging risk in Mayotte must be taken into account and must be actively monitored. In this context, collaborative work with the emergency services must continue in parallel with targeted prevention measures.

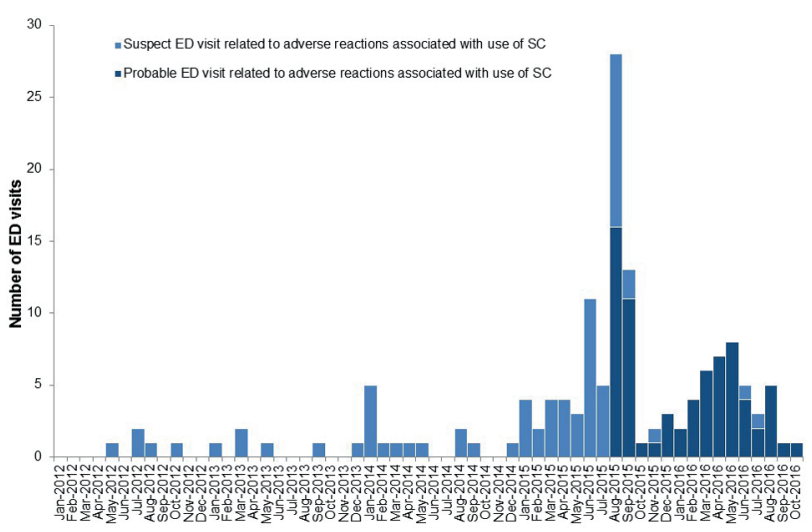

Epidemic curve of ED visits related to adverse reactions associated to use of synthetic cannabinoids, Mayotte Island, January 1st 2012 to October 30, 2016

\section{Keywords}

syndromic surveillance; synthetic cannabinoid; outbreak

\section{Acknowledgments}

We thank all healthcare workers of emergency departments of Mayotte

\section{References}

1. Vilain P, Maillard O, Raslan-Loubatie J, Abdou MA, Lernout T, Filleul L. Usefulness of Syndromic Surveillance for Early Outbreak Detection in Small Islands: The Case of Mayotte. Online Journal of Public Health Informatics. 2013;5(1):e149.

\section{*Pascal Vilain}

E-mail: pascal.vilain@ars.sante.fr 